\title{
Ein lineares Programmierungsverfahren
}

\author{
Von
}

\section{Dietmar Onigkeit}

\section{Inhaltsverzeichnis}

1. Einleitung und Beschreibung des Verfahrens . . . . . . . . . . . . . . . 82

2. Das Lösungsverfahren . . . . . . . . . . . . . . . . . . . . . . . . . . . 86

2.1 Berechnung einer zulässigen Näherung . . . . . . . . . . . . . . . . . . . 87

2.2 Eindimensionale Verbesserung . . . . . . . . . . . . . . . . . . . . . . . 89

2.3 Transformation . . . . . . . . . . . . . . . . . . . . . . 92

2.4 Optimalitätskriterium . . . . . . . . . . . . . . . . . . . . 95

2.5 Konvergenz . . . . . . . . . . . . . . . . . . . . . . . . . . . . . 96

3. Sonderfälle . . . . . . . . . . . . . . . . . . . . . . . . . . . . . . 99

3.1 Degeneration . . . . . . . . . . . . . . . . . . . . . . . . . . . . . . 99

3.2 Unendliche Lösung . . . . . . . . . . . . . . . . . . . . . . . . . 101

3.3 Mehrfache Lösung . . . . . . . . . . . . . . . . . . . . . . . . . . 103

3.4 Existenz einer Lösung . . . . . . . . . . . . . . . . . . . . . . . . . . . . 105

3.5 Normalform . . . . . . . . . . . . . . . . . . . . . . 106

\section{Einleitung und Beschreibung des Verfahrens}

Die Anwendung mathematischer Methoden hat in den letzten Jahrzehnten auch außerhalb der Naturwissenschaften sehr zugenommen. Besonders stark sind die Wirtschaftswissenschaften daran beteiligt, in denen sich hieraus neue Disziplinen entwickelten, wie z. B. die Ökonometrie in der Nationalökonomie und Operations Research in der Betriebswirtschaft. Diese Zweige haben wieder anregend auf die Mathematik gewirkt und neue Forschungsgebiete, wie z. B. Spieltheorie, Informationstheorie, Theorie der Warteschlangen, mathematische Programmierung und andere mehr in den Vordergrund gerückt. Alle diese Bereiche stehen noch mitten in der Entwicklung, und es bedarf weiterhin umfangreicher theoretischer Forschung, ehe die von der Praxis gestellten Probleme mit der gewünschten Allgemeinheit gelöst werden können.

Unter den oben erwähnten Gebieten ist die mathematische Programmierung, ihrer mannigfaltigen Anwendbarkeit wegen, von großer Bedeutung. Es handelt sich dabei um ein Extremalproblem mit Nebenbedingungen, welche in der Form von Ungleichungen gegeben sind. Die allgemeinste Formulierung würde lauten:

Bestimme ein $n$-Tupel reeller Zahlen, $x=\left(x_{1}, \ldots, x_{n}\right)$, welches die reellwertige Zielfunktion $Z(x)$ zum Optimum (Maximum oder Minimum) macht, wobei der Wertevorrat der $x$ durch ein System von Ungleichungen der Form

gegeben ist.

$$
U_{i}(x) \geqq 0 \quad(i=1,2, \ldots, m)
$$

Jedes $n$-Tupel, welches $Z(x)$ zum Optimum macht, wird als eine Lösung des Problems bezeichnet. Ein $n$-Tupel, das dem Ungleichungssystem $U_{i}(x) \geqq 0$ genügt, heißt zulässige Näherung (feasible solution). 
In dieser Allgemeinheit kann das Problem noch nicht gelöst werden. Dagegen existieren iterative Lösungsverfahren für den linearen Fall, d. h. für den Fall, daß $Z(x)$ und $U_{i}(x)$ lineare Funktionen sind.

In der Anwendung der mathematischen Programmierung kann man bei den meisten Aufgaben die Zusammenhänge in linearer Form erfassen. Dies rechtfertigt es, auch weiterhin nach Verfahren Ausschau zu halten, die es erlauben, die Lösungsmethoden zu rationalisieren. Einen Beitrag in dieser Richtung soll das hier beschriebene Programmierungsverfahren liefern. Dabei werden die folgenden Gesichtspunkte im Vordergrund stehen.

1. Eine Lösung soll, falls sie existiert, in endlich vielen Schritten erreicht werden.

2. Die Anzahl der Transformationen *, welche zur Berechnung des exakten Lösungswertes notwendig sein werden, soll möglichst klein gehalten werden.

3. Das Verfahren soll gestatten, die verfügbaren Vorkenntnisse über die ungefähre Lage einer Lösung für die Wahl einer , günstigen“ “ Ausgangsnäherung auszunutzen.

4. Es wird darauf Wert gelegt, daß das Verfahren auch beim Auftreten der sogenannten Sonderfälle (Degeneration, Fehlen einer zulässigen Ausgangsnäherung usw.) zum Ziele führt.

Die Forderung, gerade diese Punkte zu beachten, lag nahe, weil sie bei den gebräuchlichen Verfahren zum Teil nicht erfüllt sind und sich dort negativ auswirken. So gelangt man z. B. beim Gradientenverfahren von Rosex in endlich vielen Schritten im allgemeinen nur zu einer Approximation der Lösung. Das Simplexverfahren andererseits erlaubt es nicht, die oft vorhandenen Kenntnisse der ungefähren Lage einer Lösung zur Wahl einer günstigen Ausgangsnäherung heranzuziehen, weil dort in jedem Falle mit dem Koordinatenursprung als 1. Näherung begonnen werden muß. Da weiter der Lösungsweg beim Simplexverfahren nur über benachbarte Eckpunkte des durch $U_{i}(x) \geqq 0$ gegebenen Bereiches zum Lösungspunkt führt und jeder Übergang zu einem neuen Eckpunkt mit einer Transformation verbunden ist, wird die Anzahl der Transformationen recht hoch sein. Bei umfangreichen Programmierungssystemen besteht daher die Gefahr, daß der Einfluß der durch die Transformationen entstehenden Abrundungsfehler auf das Ergebnis unerwünscht groß werden kann.

Das hier beschriebene Verfahren kann nicht völlig auf die Durchführung von Transformationen verzichten. Es wird aber im allgemeinen mit weniger Transformationen auskommen als das Simplexverfahren, da für die Berechnung von verbesserten Näherungen so weit als möglich Gradientenmethoden benutzt werden. Doch lassen sich auch spezielle Beispiele konstruieren, in denen die Anzahl der Transformationen für die gleiche Ausgangsnäherung beim Simplexverfahren kleiner ist. Als Folge von 3. wird man aber bei den Anwendungen oft mit günstigeren Ausgangsnäherungen als dem Koordinatenursprung starten können.

* Im Text zur Fig. 2 ist $Z$ weck und Wirkung der Transformationen erläutert, während man ihre algebraische Darstellung in Abschnitt 2.3 findet. 
Als nächstes soll der Gedankengang des Verfahrens erläutert werden. Gegeben sei also das folgende lineare Programmierungssystem, in dem $a_{n}$ und $b_{n+i, m}$ reelle und $n, m$ und $i$ natïrliche Zahlen sind mit $1 \leqq i \leqq m$ :

Minimiere die lineare Zielfunktion

$$
Z(x)=a_{1} x_{1}+a_{2} x_{2}+\cdots+a_{n} x_{n}+a_{0}
$$

unter den linearen Nebenbedingungen

und

$$
U_{j}(x)=x_{j} \geqq 0 \quad(j=1, \ldots, n)
$$

$$
U_{n+i}(x)=b_{n+i, 1} x_{1}+\cdots+b_{n+i, n} x_{n}+b_{n+i, 0} \geqq 0 \quad(i=1, \ldots, m)
$$

Die durch $U_{j}(x)$ und $U_{n+i}(x)$ definierten Nebenbedingungen geben den zulässigen Wertbereich von $x=\left(x_{1}, \ldots, x_{n}\right)$ an (vgl. z. B. Fig. 1). Diejenigen. Werte $n$-tupel $y_{\text {opt }}=\left(y_{1}, \ldots, y_{n}\right)$ aus dem oben definierten Bereich, welche die Zielfunktion $Z$ zum Minimum machen, sind dann die gesuchten Lösungen.

Angenommen man kenne eine zulässige Näherung. Diese Bedingung erfüllt

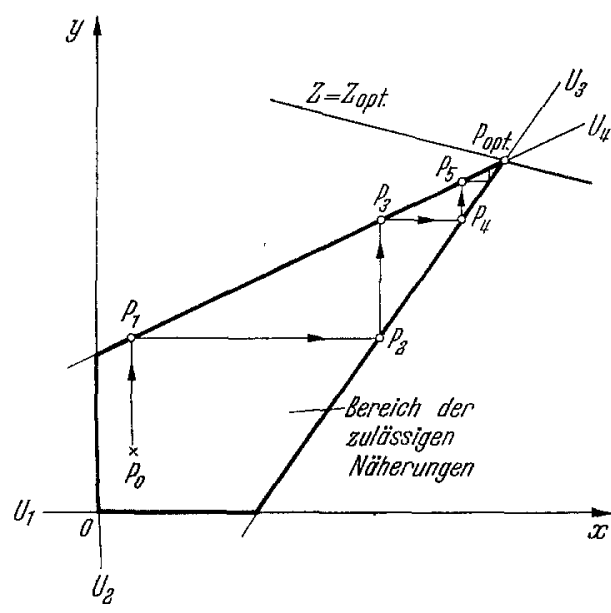

Fig. 1 z. B. der Punkt $P_{0}$ in Fig. 1. Von $P_{0}$ ausgehend, wird man dann versuchen, durch Änderung des Wertes nur einer Variablen (z.B. von $y$ ) zu einer verbesserten Näherung $P_{1}$ zu gelangen. Diese muß dann ebenfalls eine zulässige Näherung sein und - in die Zielfunktion eingesetzt - einen kleineren Wert ergeben, als die Ausgangsnäherung.

Man sagt, falls beides erfüllt ist, man sei durch eindimensionale Verbesserung zu einer verbesserten Näherung gelangt. Natürlich wird man bei der eindimensionalen Verbesserung den Wert derjenigen Variablen ändern, die eine möglichst große Verminderung der Zielfunktion bewirkt.

Mit der eben erhaltenen verbesserten Näherung als neuer Ausgangsnäherung kann man versuchen, die eindimensionale Verbesserung erneut anzuwenden. Fährt man so fort, dann können zwei Fälle auftreten.

1. Die eindimensionale Verbesserung ist beliebig oft wiederholbar. Aus dem Beispiel der Fig. 1 ist es unmittelbar ersichtlich, daß man durch eindimensionale Verbesserung nur in unendlich vielen Schritten vom Ausgangspunkt $P_{0}$ zur Lösung $P_{\text {opt }}$ gelangen kann. Diese Schwierigkeit wird man vermeiden, indem man das Verfahren der eindimensionalen Verbesserung an geeigneter Stelle abbricht und einen anderen Weg einschlägt. Als Kriterium dafür kann z. B. derjenige Zeitpunkt gewählt werden, an dem die verbesserte Näherung eine der Ungleichungen zum 3. Male als Gleichung erfüllt. In Fig. 1 geschieht das im Punkte $P_{5}$.

2. Die eindimensionale Verbesserung ist nur endlich viele Male wiederholbar. 
Im Beispiel der Fig. 2 ist von der Ausgangsnäherung $P_{0}$ nur eine eindimensionale Verbesserung zum Punkte $P_{1}$ hin möglich. Denn der Weg von $P_{1}$ nach $P_{2}$ erfordert die gleichzeitige Änderung der Variablen $x$ und $y$.

Nach Konstruktion liegt jede verbesserte Näherung auf dem Rande des zulässigen Bereiches, falls dieser abgeschlossen ist. Die Näherung $P_{1}$ erfüllt z. B. die Ungleichung $U_{1}$ exakt, d.h. sogar als Gleichung.

Aus Fig. 2 ergibt sich unmittelbar das weitere Vorgehen. Man wird von $P_{1}$ aus durch gleichzeitige Änderung beider Koordinaten versuchen, längs $U_{1}$ zur Näherung $P_{2}$ zu gelangen. Die geplante zweidimensionale Verbesserung läßt sich aber leicht auf eine eindimensionale zurückführen. Den Vorteil dieser Rückführung erkennt man sofort, wenn man z. B. in einem

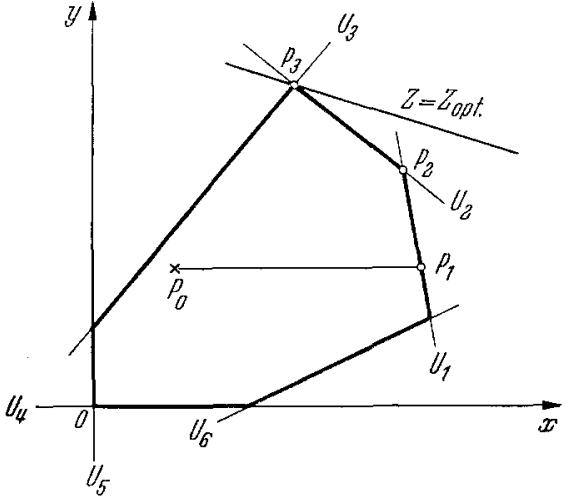

Fig. 2 $n$-dimensionalen Programmierungssystem statt durch gleichzeitige Änderung aller $n$ Variablen nur durch die Änderung einer Variablen eine bessere Näherung zu suchen hat. Die Rückführung auf eine eindimensionale Verbesserung wird mit Hilfe von Transformationen erreicht.

Dazu löst man die durch die Näherung $P_{1}$ exakt erfüllte Ungleichung $U_{1}$ nach einer geeigneten Variablen auf, setzt sie in das Programmierungssystem ein und reduziert dieses dadurch um eine Variable und eine Ungleichung. Jeder eindimensionalen Verbesserung des reduzierten Programmierungssystems entspricht jetzt im ursprünglichen System eine zweidimensionale Verbesserung längs des durch $U_{1}$ gegebenen Randes.

Nun sieht man aber aus Fig. 2, daß die optimale Lösung $P_{3}$ nicht auf $U_{1}$ liegt, während als Folge der Transformation alle weiteren Näherungen $U_{1}$ exakt erfüllen müssen. Diese Schwierigkeit wird am besten umgangen, indem man die exakt erfüllte Ungleichung $U_{1}$ noch vor der Transformation durch Subtraktion einer Hilfsvariablen z zu $\overline{U_{1}}$ erweitert. Die zur Transformationsgleichung erweiterte Ungleichung $U_{1}$ nimmt dann nach der Transformation die Form $z \geqq 0$ an. Damit ist dafür gesorgt, daß durch die Wahl von $z$ der zulässige Bereich der Nebenbedingungen nicht vergrößert werden kann. Dies erkennt man sofort, weil die erweiterte Ungleichung $\bar{U}_{1}$ für $z=0$ identisch mit $U_{1}$ ist und für alle $z>0$ Parallelen zu $U_{1}$ liefert, die den zulässigen Bereich höchstens einengen können. Erhält man also als Folge einer späteren eindimensionalen Verbesserung Werte $z>0$, dann kann die verbesserte Näherung nicht mehr auf $U_{1}$ liegen.

Der oben erklärte Vorgang der Transformation, der im Abschnitt 2.3 ausführlich dargestellt wird, läßt sich nun wie folgt zusammenfassen.

Von der zuletzt erhaltenen Näherung wird mindestens eine der Ungleichungen exakt erfüllt. Diese ist durch Subtraktion einer Hilfsvariablen zu erweitern und nach einer geeigneten Variablen, der Transformationsvariablen, aufzulösen. Die so erhaltene Gleichung wird Transformationsgleichung genannt. Durch Einsetzen der 
Transformationsvariablen in das Programmierungssystem erhält man das transformierte Programmierungssystem.

Jeder Näherung des transformierten Systems ist eineindeutig eine Näherung des ursprünglichen Systems zugeordnet. Da überdies gezeigt werden kann, daß auch jeder Lösung des ursprünglichen Systems eineindeutig eine Lösung des transformierten Systems zugeordnet ist, darf man mit dem letzteren weiterrechnen. Man wird also versuchen, erneut die eindimensionale Verbesserung anzuwenden und im oben beschriebenen Sinne fortfahren, bis man eine Lösung erreicht hat.

Dieser Sachverhalt läßt sich gut veranschaulichen, wenn man die Transformation als Koordinatentransformation interpretiert. An Hand des Beispiels von Fig.2

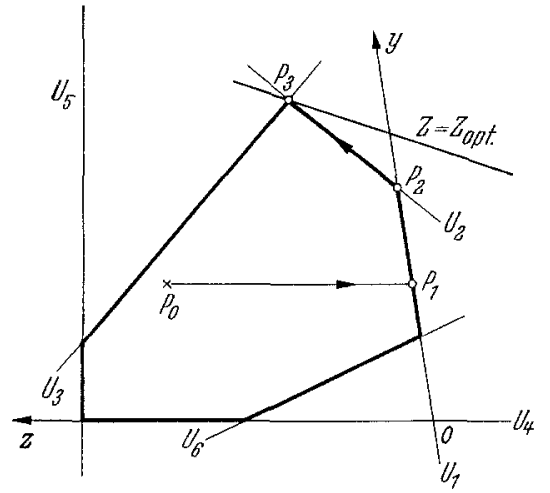

Tig. 3 soll dies in Fig. 3 geschehen. Wählt man z. B. $x$ als Transformationsvariable aus, dann wird das neue Koordinatensystem durch die Geraden $y=0$ und $z=0$ des alten Systems gebildet. An Stelle der bisherigen Koordinatenachse $U_{5}$ oder $x=0$ ist jetzt die Gerade $U_{1}$ oder $z=0$ Koordinatenachse geworden. Das Programmierungssystem selbst ist aber unverändert geblieben, abgesehen von der Einführung eines anderen Koordinatensystems und der entsprechenden Änderung in der Bezeichnung.

Aus Fig. 3 ist ferner ersichtlich, daB im Gegensatz zum Koordinatensystem in Fig. 2 - von der Näherung $P_{1}$ aus eine eindimensionale Verbesserung durch Änderung des $y$-Wertes vorgenommen werden kann. Diese führt zur verbesserten Näherung $P_{2}$, die in Fig. 2 nur durch eine zweidimensionale Verbesserung erhalten werden konnte.

Mit der Näherung $P_{2}$ als Ausgangsnäherung hat man dann weiter zu prüfen, ob wieder eine eindimensionale Verbesserung möglich ist. Im obigen Beispiel ist das nicht der Fall. Also führt man nochmals eine Transformation durch mit $y$ als Transformationsvariablen und $\bar{U}_{2}$ als Transformationsgleichung. So gelangt man durch wiederholte Anwendung von eindimensionaler Verbesserung und Transformation von der zulässigen Ausgangsnäherung $P_{0}$ zur Lösung $P_{\mathbf{3}}$.

\section{Das Lösungsverfahren}

Ein lineares Programmierungssystem läßt sich wie im Abschnitt 3.5 gezeigt wird, auf die folgende Normalform bringen.

Minimiere die Zielfunktion

$$
Z(x)=a_{1} x_{1}+a_{2} x_{2}+\cdots+a_{n} x_{n}+a_{0}
$$


unter den Nebenbedingungen

und

$$
\begin{array}{llll}
U_{1}(x)=x_{1} & & & \geqq 0 \\
U_{2}(x)= & & x_{2} & \geqq 0
\end{array}
$$

$$
U_{n}(x)=\quad x_{n} \geqq 0
$$

$$
\begin{aligned}
& U_{n+1}(x)=b_{n+1,1} x_{1}+\cdots+b_{n+1, n} x_{n}+b_{n+1,0} \geqq 0 \\
& U_{n+2}(x)=b_{n+2,1} x_{1}+\cdots+b_{n+2, n} x_{n}+b_{n+2,0} \geqq 0 \\
& \ldots \ldots \ldots \ldots \ldots \ldots \ldots \ldots \ldots \ldots \ldots \ldots \ldots \ldots \ldots \\
& U_{n+m}(x)=b_{n+m, 1} x_{1}+\cdots \ldots+b_{n+m, n} x_{n}+b_{n+m, 0} \geqq 0
\end{aligned}
$$

Die erste Näherung wird willkürlich angenommen. Sie sei z. B.

$$
y=\left(y_{1}, y_{2}, \ldots, y_{j}, \ldots, y_{n}\right)
$$

Setzt man $y$ in die Ungleichungen (2) und (3) ein, so können zwei Fälle eintreten.

1. Die Näherung (4) ist eine zulässige Näherung, d. h. $y$ erfüllt alle Ungleichungen in (2) und (3).

2. $y$ ist keine zulässige Näherung.

Der 2. Fall läßt sich mit Hilfe der lincaren Programmierung auf den 1. Fall zurückführen. Dios behandelt der folgende Abschnitt*.

\subsection{Berechnung einer zulässigen Näherung}

Hier soll gezeigt werden, wie man eine Näherung erhalten kann, die keine der Nebenbedingungen des Programmierungssystems verletzt.

Es seien - nötigenfalls nach geeigneter Umordnung -

$$
\begin{aligned}
& U_{1}(y)=b_{11} y_{1}+\cdots+b_{1 j} y_{j}+\cdots+b_{1 n} y_{n}+b_{10} \geqq 0 \\
& \ldots \ldots \ldots \ldots \ldots \ldots \ldots \\
& U_{r}(y)=b_{r 1} y_{1}+\cdots+b_{r j} y_{j}+\cdots+b_{r n} y_{n}+b_{r 0} \geqq 0
\end{aligned}
$$

$(0 \leqq r \leqq m+n)$ die von (4) erfüllten Ungleichungen aus (2) und (3) und

$$
\begin{aligned}
& U_{1}^{*}(y)=b_{11}^{*} y_{1}+\cdots+b_{1 j}^{*} y_{j}+\cdots+b_{1 n}^{*} y_{n}+b_{10}^{*}<0 \\
& \cdots \cdots \cdots \cdots \\
& U_{s}^{*}(y)=b_{s 1}^{*} y_{1}+\cdots+b_{s j}^{*} y_{j}+\cdots+b_{s n}^{*} y_{n}+b_{s 0}^{*}<0
\end{aligned}
$$

mit $s=m+n-r$ die von (4) nicht erfüllten Ungleichungen. Da angenommen wird, daß $y$ lkeine zulässige Näherung ist, gilt $s \geqq 1$.

* Das hier beschriebene Vorgehen entspricht der Idee der Mehrphasenmethode. Vgl. KüNzr, H. P. „Die Simplexmethode zur Bestimmung einer Ausgangslösung bei bestimmten linearen Programmen“. Unternehmensforschung 2, 60-69 (1958). 
Jetzt addiert man die mit -1 multiplizierten Relationen (6) und erhält

$$
W^{*}(y)=B_{1}^{*} y_{1}+\cdots+B_{j}^{*} y_{j}+\cdots+B_{n}^{*} y_{n}+B_{0}^{*}>0,
$$

wenn man die Abkürzungen

$$
W^{*}=-\sum_{\alpha=1}^{s} U_{\alpha}^{*} \text { und } B_{h}^{*}=-\sum_{\alpha=1}^{s} b_{\alpha h}^{*}, \quad(h=0,1, \ldots, n)
$$

benutzt.

Findet man nun eine Näherung $y^{\prime}$, die alle Ungleichungen (5) erfüllt und für die

$$
W^{*}\left(y^{\prime}\right) \leqq 0
$$

gilt, dann wird für mindestens eine der Ungleichungen (6) $U_{\alpha}^{*}\left(y^{\prime}\right) \geqq 0(1 \leqq \alpha \leqq s)$ gelten. Denn wäre dies nicht der Fall, so würden alle $U_{\alpha}^{*}\left(y^{\prime}\right)<0$ sein und damit

$$
W^{*}\left(y^{\prime}\right)=-\sum_{\alpha=1}^{s} U_{\alpha}^{*}\left(y^{\prime}\right)>0
$$

gelten. Das widerspräche aber der Voraussetzung (9).

Existiert kein $y^{\prime}$, welches neben (5) auch (9) erfüllt, dann gibt es keine zulässige Näherung und folglich auch keine Lösung für (1) bis (3).

Nun wird durch eine zusätzliche Bedingung dafür gesorgt, daß sich $y^{\prime}$ nicht ,unnötig weit" von einer Lösung entfernen kann.

Erhält man z. B. $y^{\prime}$ aus der Relation.

$$
y^{\prime}=\left(y_{1}, \ldots, y_{j-1}, y_{j}+\Delta y_{j}, y_{j+1}, \ldots, y_{n}\right),
$$

dann soll für möglichst viele Ungleichungen von (6)

$$
U_{\alpha}^{*}\left(y^{\prime}\right)=U_{\alpha}^{*}(y)+b_{\alpha j}^{*} \Delta y_{j} \geqq 0
$$

sein. Bezeichnet man die positiven $b_{\alpha j}^{*}$ mit $b_{\alpha j}^{*+}$ und die negativen $b_{\alpha j}^{*}$ mit $b_{\alpha j}^{*}$, so wird diese Forderung erfüllt, wenn man entweder

$$
\Delta y_{j}=\max _{\alpha}\left(-\frac{U_{\alpha}^{*}(y)}{b_{\alpha j}^{*+}}\right)
$$

oder

$$
\Delta y_{j}=\min _{\alpha}\left(-\frac{U_{\alpha}^{*}(y)}{b_{\alpha i}^{*-}}\right)
$$

setzt.

Jede Wahl von $\Delta y_{j}$ über die durch

$$
\min _{\alpha}\left(-\frac{U_{\alpha}^{*}(y)}{b_{\alpha, j}^{*-}}\right) \leqq \Delta y_{j} \leqq \max _{\alpha}\left(-\frac{U_{\alpha}^{*}(y)}{b_{\alpha j}^{*+}}\right)
$$

gegebenen Grenzen* hinaus, bringt keinen weiteren Vorteil. Denn wählt man z. B. $\Delta y_{j}>\max \left(-U_{\alpha}^{*}(y) / b_{\alpha j}^{*}\right)$, so kann $y^{\prime}$ nicht mehr Ungleichungen von (6) erfüllen, als wenn $\triangle y_{j}=\max _{\alpha}\left(-U_{\alpha}^{*}(y) / b_{\alpha j}^{*+}\right)$ wäre.

$\star$ Existiert kein $b_{\alpha i}^{*+}$, so lautet $(11): \min \left(-U_{\alpha}^{*}(y) / b_{\alpha j}^{*-}\right) \leqq \Delta y_{j}$. Analoges gilt, wenn kein $b_{\alpha i j}^{*-}$ existiert. 
Durch (11) ist also sichergestellt, daß der Betrag von $\Delta y_{j}$ nicht mehr vergrößert wird, wenn dadurch keine weitere Ungleichung aus (6) größer oder gleich Null werden kann.

Kennt man nun zur Bestimmung von $y^{\prime}$ ein Verfahren, welches unter Berücksichtigung von (5) und (11) die Relation (9) erfüllt, dann ist mit $y^{\prime}$ eine Näherung von (1) bis (3) gewonnen, die mindestens eine Ungleichung mehr erfüllt als $y$. Durch nochmalige Anwendung des Verfahrens mit $y^{\prime}$ als Ausgangsnäherung und einer nach (8) neu errechneten Relation $W^{* *}$ für (9) erhält man eine weitere Näherung $y^{\prime \prime}$, die wieder mehr Ungleichungen erfüllt als $y^{\prime}$. So fährt man fort, bis man nach spätestens $s$ Wiederholungen zu einer zulässigen Näherung - ihre Existenz vorausgesetzt - gekommen ist.

Als Verfahren zur Bestimmung von $y^{\prime}$ kann nun die lineare Programmierung herangezogen werden. Denn bestimmt man $y^{\prime}$ so, daB (7) unter den Nebenbedingungen (5) und (11) minimal wird, dann ist - falls (1) bis (3) lösbar ist - auch die Bedingung (9) erfüllt.

Es erweist sich als vorteilhaft, für jede nach (10) erhaltene verbesserte Näherung $y^{\prime}$ zu prüfen, ob für eine der Ungleichungen von (6) $U_{\alpha}^{*}\left(y^{\prime}\right) \geqq 0$ gilt. Ist das der Fall, so wird man für $y^{\prime}$ als Ausgangsnäherung (5) und (7) neu zusammenstellen und die Programmierung von vorne beginnen. Dadurch wird verhindert, daß nach einer weiteren "Verbesserung" wieder $U_{\alpha}^{*}\left(y^{\prime \prime}\right)<0$ gelten kann.

Damit ist gezeigt, wie man mit Hilfe der linearen Programmierung zu einer zulässigen Näherung kommen kann. Die Bedingung (11) entspricht zwar nicht der Formulierung der linearen Programmierung in (1) bis (3), ist dafür aber so gewählt, daß sie sich mühelos in die Schematik des Programmierungsverfahrens einordnen läßt.

\subsection{Eindimensionale Verbesserung}

Gegeben ist das lineare Programmierungssystem (1) bis (3) und die willkürlich angenommene Näherung (4).

Jetzt soll untersucht werden, ob (4) durch die Änderung $\Delta y_{j}$ in nur einer Variablen verbessert werden kann. Durch diese eindimensionale Verbesserung würde man eine neue Näherung

$$
y^{\prime}=\left(y_{1}, \ldots, y_{j-1}, y_{j}+\Delta y_{j}, y_{j+1}, \ldots, y_{n}\right)
$$

erhalten, wobei $y^{\prime}$ den folgenden Voraussetzungen zu entsprechen hätte:

I. Keine der von $y$ bereits erfüllten Ungleichungen $U_{i}$ darf durch $y^{\prime}$ verletzt werden.

II. $y^{\prime}$ soll eine bessere Näherung sein als $y$. D. h., falls $y$ eine zulässige Näherung ist, hat $Z\left(y^{\prime}\right)<Z(y)$ zu gelten. Wenn dagegen $y$ keine zulässige Näherung ist, so wird nach Abschnitt 2.1 die Rolle der Zielfunktion von (7) eingenommen, und es muß daher $W^{*}\left(y^{\prime}\right)<W^{*}(y)$ sein. 
Nun soll die Voraussetzung I untersucht werden. Erfüllt $y$ die Ungleichung $U_{i}(y) \geqq 0(i=1, \ldots, r)$, dann soll auch

$$
U_{i}\left(y^{\prime}\right)=U_{i}(y)+b_{i j} \Delta y_{j} \geqq 0
$$

sein. Das ist der Fall, wenn

für $b_{i j}^{+}\left(\right.$d. h. $\left.b_{i j}>0\right)$

und für $b_{i j}^{-}$(d. h. $b_{i j}<0$ )

$$
-\frac{U_{i}(y)}{b_{i j}^{+}} \leqq \Delta y_{j}
$$

$$
\Delta y_{j} \leqq-\frac{U_{i}(y)}{b_{i j}^{-}}
$$

gilt. Alle Ungleichungen $U_{i}$ mit $b_{i j}=0$ können bei der Berechnung von $\Delta y_{j}$ fortgelassen werden, da $\Delta y_{j}$ ihren Wert nicht ändern kann.

Faßt man die obigen, für jede einzelne Ungleichung $U_{i}$ gültigen Relationen zusammen, dann erhält man als Variationsbereich* für $\Delta y_{j}$

$$
\max _{i}\left(-\frac{U_{i}(y)}{b_{i j}^{+}}\right) \leqq \Delta y_{j} \leqq \min _{i}\left(-\frac{U_{i}(y)}{b_{i j}}\right)
$$

Genügt also $\Delta y_{j}$ der Bedingung (12), dann gilt mit $U_{i}(y) \geqq 0$ auch stets $U_{i}\left(y^{\prime}\right) \geqq 0$.

Die Voraussetzung II verlangt, daß

bzw.

$$
Z\left(y^{\prime}\right)=Z(y)+\Delta Z=Z(y)+a_{j} \Delta y_{j}<Z(y)
$$

wird.

$$
W^{*}\left(y^{\prime}\right)=W^{*}(y)+\Delta W^{*}=W^{*}(y)+B_{j}^{*} \Delta y_{j}<W^{*}(y)
$$

Sollte kein $\Delta y_{j}$, das der Voraussetzung $I$ genügt, die obige Forderung erfüllen, dann ist eine eindimensionale Verbesserung nicht möglich. Für diesen Fall wird das weitere Vorgehen im Abschnitt 2.3 beschrieben.

Es kann daher angenommen werden, daß in (12) mindestens ein $\Delta y_{j}$ existiert, welches die obige Relation erfüllt. Jetzt versucht man natürlich, das günstigste dieser $\Delta y_{j}$ für die eindimensionale Verbesserung heranzuziehen.

Wenn $y$ eine zulässige Näherung ist, wird also stets jenes $\Delta y_{j}$ aus (12) ausgewählt, das $\Delta Z=a_{j} \Delta y_{j}$ möglichst klein werden läßt. Dies ergibt für die optimale Verbesserung der Zielfunktion

mit

$$
\Delta Z=\min _{j} a_{j} \Delta y_{j}
$$

$$
\Delta y_{j}= \begin{cases}\max _{i}\left(-\frac{U_{i}(y)}{b_{i j}^{+}}\right) & \text {für } a_{j}>0 \\ \min _{i}\left(-\frac{U_{i}(y)}{b_{\bar{i}}^{-}}\right) & \text {für } a_{j}<0\end{cases}
$$

* Ist $L$ die leere Menge, so möge gelten: $\max (L)=-\infty$ und $\min (L)=+\infty$. Gilt also für (12) $-\infty \leqq \Delta y_{j} \leqq+\infty$, dann soll darunter verstanden werden, daß die Wahl von $\Delta y_{j}$ keinen Beschränkungen unterworfen ist. 
Ist $y$ keine zulässige Näherung, so soll durch $\Delta y_{j}$ der Wert von $\Delta W^{*}=B_{j}^{*} \Delta y_{j}$ möglichst klein werden. Berücksichtigt man aus den in 2.2 erklärten Gründen noch die Relation (11), dann erhält man analog als optimale Verbesserung

$$
\Delta W^{*}=\min _{j} B_{j}^{*} \Delta y_{j}
$$

mit

$$
\Delta y_{j}=\left\{\begin{array}{lll}
\max _{i}\left(-\frac{U_{i}(y)}{b_{i j}^{+}},\right. & \left.\min _{\alpha \alpha}-\frac{U_{\alpha}^{*}(y)}{b_{\alpha i}^{*}}\right) & \text { für } B_{j}^{*}>0 \\
\min _{i}\left(-\frac{U_{i}(y)}{b_{\bar{i}}^{-}},\right. & \left.\max _{\alpha}-\frac{U_{\alpha}^{*}(y)}{b_{\alpha i}^{*+}}\right) & \text { für } B_{j}^{*}<0
\end{array}\right.
$$

Durch die eindimensionale Verbesserung nach (13) und (14) bzw. (13*) und (14*) gelangt man zur verbesserten Näherung $y^{\prime}$. Jetzt kann man mit $y^{\prime}$ als Ausgangsnäherung (4) versuchen, diesen Prozeß zu wiederholen. Sollte die eindimensionale Verbesserung beliebig oft wiederholbar sein, so ist es, wie Fig. 1 zeigt, notwendig, das Verfahren an geeigneter Stelle abzubrechen, um in endlich vielen Schritten zu einer Lösung ${ }^{\star}$ gelangen zu können ${ }^{\star \star}$. Dann wird man durch geeignete Transformationen dafür sorgen, daß sich die eindimensionale Verbesserung erfolgreich fortsetzen läßt. Dies behandelt der nächste Abschnitt.

Die Entscheidung, wann die eindimensionale Verbesserung nicht weiter fortgesetzt werden, sondern durch Transformationen unterbrochen werden soll, fällt man mit Hilfe des

Transformationskriteriums: An die Stelle weiterer eindimensionaler Verbesserungen tritt eine Transformation, wenn entweder wegen

$$
\Delta Z=0 \text { bzw. } \Delta W^{*}=0
$$

keine eindimensionale Verbesserung mehr möglich ist, oder eine der verbesserten $N \ddot{a}$ herungen eine der Ungleichungen zum r. Male exakt erfüllt. $(r>0$, ganz z.B. $r=3$.)

Die Wahl der letzten Bedingung ist willkürlich ${ }^{\star \star \star}$, hat sich jedoch bei der Behandlung der Sonderfälle als zweckmäßig erwiesen.

* Eine zulässige Näherung $y$ von (1) bis (3) wird endliche Lösung genannt, wenn für jede andere zulässige Näherung $y^{\prime}$ die Relation

erfüllt ist.

$$
Z\left(y^{\prime}\right) \geqq Z(y)
$$

$\star \star$ In $n$ dimensionalen Beispielen mit $n>2$ kann bei unendlich oft wiederholter eindimensionaler Verbesserung die Näherung sogar gegen einen Punkt konvergieren, der keine Lösung darstellt.

$\star \star \star$ Die auf den ersten Blick naheliegende Festsetzung des Transformationskriteriums als

$$
|\Delta Z| \leqq D \text { bzw. }\left|\Delta W^{*}\right| \leqq D, D>0
$$

erweist sich als unzweckmäßig. Denn führt man $\left(15^{*}\right)$ statt (15) als Transformationskriterium ein, dann erreicht man z. B. beim linearen Programmierungssystem: ,Minimiere $Z=-x-y$ unter den Nebenbedingungen $x, y \geqq 0$ und $x-y \geqq-D / 3$, sowie $y-x \geqq-D / 3$ " eine unendliche Lösung erst nach unendlich vielen Schritten.

Auch zur Wahl von $r$ soll noch ein Hinweis gegeben werden. Setzt man $r=1$, so ist - mit einem Eckpunkt des durch (2) und (3) gegebenen Bereiches als Ausgangsnäherung - jede verbesserte Näherung analog dem Simplexverfahren ein benachbarter Eckpunkt. Die Anzahl der Transformationen würde dadurch unnötig groß werden. Wählt man dagegen für $r$ eine große ganze Zahl, so macht man in gewissen Fällen (vgl. Fig. 1) eine unnötig große Anzahl von eindimensionalen Verbesserungen. In dieser Hinsicht ist die Wahl von $r=2$ oder 3 zweckmäßig. 


\subsection{Transformation}

Es kann hier angenommen werden, daß eine Wiederholung der eindimensionalen Verbesserung aus den in 2.2 genannten Gründen nicht möglich ist. Dann lassen sich geeignete Transformationen finden, die es erlauben, die eindimensionale Verbesserung wieder fortzusetzen. Durch wechselweise Anwendung der eindimensionalen Verbesserung und der Transformation kann man in endlich vielen Schritten zu einer Lösung gelangen.

In diesem Abschnitt wird die entsprechende Transformationsgleichung aus den. Eigenschaften, denen sie genügen muß, abgeleitet. Dazu wird vorausgesetzt, daß keine der verbesserten Näherungen mehr als $n$ Ungleichungen exakt erfüllt, d. h., daß keine Degeneration auftritt. Außerdem kann man sich im folgenden auf die Erläuterung am System (1) bis (3) beschränken, weil die Lösung von (7) und (5) mit Ausnahme von (13*) und $\left(14^{*}\right)$ völlig analog verläuft.

Bei jedem Abbruch der eindimensionalen Verbesserung erfüllt die verbesserte Näherung (10) als Folge von (12) mindestens eine der Ungleichungen vom Typ (3) exakt*. Eine beliebige davon - es sei z. B. $U_{n+k}$ - wird als Transformationsgleichung herangezogen. Dazu erweitert man $U_{n+k}$ durch Addition von $b_{n+k, n+k} \times$ $\times x_{n+k} \mathrm{zu}$

$$
\bar{U}_{n+k}=b_{n+k, 1} x_{1}+\cdots+b_{n+k, n} x_{n}+b_{n+k, n+k} x_{n+k}+b_{n+k, 0}=0
$$

Hier spielt die Hilfsvariable $x_{n+k}$ die Rolle einer sogenannten Schlupfvariablen, während

$$
b_{n+k, n+k}=-1
$$

gesetzt wird.

Nun löst man $\bar{U}_{n+k}$ nach einer geeigneten Variablen $x_{g}$ auf. Aus Gründen, die in den nächsten Abschnitten ersichtlich werden, wird die Auswahl des Index $g$ wie folgt vorgenommen: Bezeichnet man mit $\{\delta\}$ die Menge aller Indizes $\mathrm{j}$, für die $b_{n+k, j} \neq 0$ gilt und mit $y=\left(y_{1}, \ldots, y_{j}, \ldots, y_{n}\right)$ die zuletzt erhaltene zulässig Näherung, dann wird durch

$$
y_{g}^{*}=\max _{\delta} y_{\delta}
$$

eine Indexmenge $\left\{g^{*}\right\}$ definiert. Jedes beliebige Element von $\left\{g^{*}\right\}$ kann als Index $g$ ausgewählt werden.

So erhält man die Transformationsgleichung

$$
x_{g}=U_{g}^{\prime}(\bar{x})
$$

mit

und

$$
\bar{x}=\left(x_{1}, \ldots, x_{g-1}, x_{g+1}, \ldots, x_{n}, x_{n+k}\right)
$$

$$
\begin{aligned}
U_{g}^{\prime}(\bar{x})= & -\frac{1}{b_{n+k, g}}\left(b_{n+k, 1} x_{1}+\cdots+b_{n+k, g-1} x_{g-1}+b_{n+k, g+1} x_{g+1}+\right. \\
& \left.+\cdots+b_{n+k, n} x_{n}+b_{n+k, n+k} x_{n+k}+b_{n+k, 0}\right)
\end{aligned}
$$

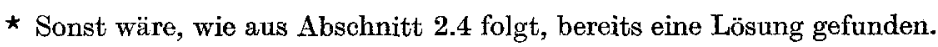


Setzt man nun (18) in das lineare Programmierungssystem (1) bis (3) ein und berücksichtigt dabei noch (16), dann erhält man das transformierte Programmierungssystem (20) bis (21).

Minimiere die Zielfunktion

$$
Z^{\prime}(\bar{x})=a_{1}^{\prime} x_{1}+\cdots+a_{g-1}^{\prime} x_{g-1}+a_{g+1}^{\prime} x_{g+1}+\cdots+a_{n}^{\prime} x_{n}+a_{n+k}^{\prime} x_{n+k}+a_{0}^{\prime}
$$

unter den Nebenbedingungen

$$
\begin{aligned}
& U_{1}^{\prime}(\bar{x}) \quad=x_{1} \quad \geqq 0 \\
& U_{g-1}^{\prime}(\bar{x})=x_{g-1} \quad \geqq 0 \\
& U_{g}^{\prime}(\bar{x}) \quad=b_{g, 1}^{\prime} x_{1}+\cdots+b_{g, g-1}^{\prime} x_{g-1}+b_{g, g+1}^{\prime} x_{g+1}+\cdots+ \\
& +b_{g, n}^{\prime} x_{n}+b_{g, n+k}^{\prime} x_{n+k}+b_{g, 0}^{\prime} \geqq 0 \\
& U_{g+1}^{\prime}(\bar{x})=x_{g+1} \quad \geqq 0
\end{aligned}
$$

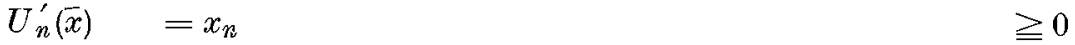

und

$$
\begin{aligned}
& U_{n+1}^{\prime}(\bar{x})=b_{n+1,1}^{\prime} x_{1}+\cdots+b_{n+1, g-1}^{\prime} x_{g-1}+b_{n+1, g+1}^{\prime} x_{g+1}+ \\
& +\cdots+b_{n+1, n}^{\prime} x_{n}+b_{n+1, n+k}^{\prime} x_{n+k}+b_{n+1,0}^{\prime} \quad \geqq 0 \\
& U_{n+k-1}^{\prime}(\bar{x})=b_{n+k-1,1}^{\prime} x_{1}+\cdots+b_{n+k-1, g-1}^{\prime} x_{g-1}+b_{n+k-1, g+1}^{\prime} x_{g+1}+ \\
& +\cdots+b_{n+k-1, n}^{\prime} x_{n}+b_{n+k-1, n+k}^{\prime} x_{n+k}+b_{n+k-1,0}^{\prime} \geqq 0 \\
& U_{n+k}^{\prime}(\bar{x})=x_{n+k} \quad \geqq 0 \\
& U_{n+k+1}^{\prime}(\vec{x})=b_{n+k+1,1}^{\prime} x_{1}+\cdots+b_{n+k+1, g-1}^{\prime} x_{g-1}+b_{n+k+1, g+1}^{\prime} x_{g+1}+ \\
& +\cdots+b_{n+k+1, n}^{\prime} x_{n}+b_{n+k+1, n+k}^{\prime} x_{n+k}+b_{n+k+1,0}^{\prime} \geqq 0 \\
& U_{n+m}^{\prime}(\bar{x})=b_{n+m, 1}^{\prime} x_{1}+\cdots+b_{n+m, g-1}^{\prime} x_{g-1}+b_{n+m, g+1}^{\prime} x_{g+1}+ \\
& +\cdots+b_{n+m, n}^{\prime} x_{n}+b_{n+m, n+k}^{\prime} x_{n+k}+b_{n+m, 0}^{\prime} \quad \geqq 0 \text {. }
\end{aligned}
$$

Dabei wurden die folgenden Abkürzungen benutzt:

$$
a_{o}^{\prime}=a_{c}-a_{g} \frac{b_{n+k, c}}{b_{n+k, g}}, \quad(c=0,1, \ldots, g-1, g+1, \ldots, n, n+k)
$$

(23) und

$$
b_{e, c}^{\prime}=b_{e, c}-b_{e, g} \frac{b_{n+k, c}}{b_{n+k, g}}, \quad(e=g, n+1, \ldots, n+k-1, n+k+1, \ldots, n+m)
$$

mit $a_{n+k}=b_{g, c}=0$ und $b_{g, g}=1$.

Um in Zukunft mit dem transformierten System weiterrechnen zu können, ist zu zeigen, wie man aus einer Lösung des transformierten Systems eine Lösung des Ausgangssystems (1) bis (3) erhalten kann.

Es sei

$$
\bar{y}=\left(y_{1}, \ldots, y_{g-1}, y_{g+1}, \ldots, y_{n}, y_{n+k}\right)
$$


eine zulässige Näherung des Systems (20) bis (22), dann ist auch

$$
y=\left(y_{1}, \ldots, y_{g-1}, y_{g}=U_{g}^{\prime}(\bar{y}), y_{g+1}, \ldots, y_{n}\right)
$$

eine zulässige Näherung von (1) bis (3). (24) und (25) werden einander zugeordnete Näherungen genannt.

Ist (24) eine Lösung von (20) bis (22), so ist (25) die zugeordnete Lösung von (1) bis (3). Der Beweis hierfür wird indirekt geführt.

Angenommen, (25) sei keine Lösung von (1) bis (3), sondern diese sei durch

$$
y^{*}=\left(y_{1}^{*}, \ldots, y_{n}^{*}\right)
$$

mit $Z\left(y^{*}\right)<Z(y)$ und $y^{*} \neq y$ gegeben. Die zu $y^{*}$ zugeordnete Näherung ist dann

$$
\bar{y}^{*}=\left(y_{1}^{*}, \ldots, y_{g-1}^{*}, y_{g+1}^{*}, \ldots, y_{n}^{*}, y_{n+k}^{*}=U_{n+k}^{*}\left(y^{*}\right)\right) \text {. }
$$

Nach Konstruktion von $Z^{\prime}$ gilt aber für die zugeordneten Näherungen $y$ und $\vec{y}$ bzw. $y^{*}$ und $\bar{y}^{*}$

$$
Z(y)=Z^{\prime}(\bar{y}) \text { bzw. } Z\left(y^{*}\right)=Z^{\prime}\left(\bar{y}^{*}\right) .^{\star}
$$

Wäre nun $Z\left(y^{*}\right)<Z(y)$, dann müßte auch

$$
Z^{\prime}\left(\bar{y}^{*}\right)=Z\left(y^{*}\right)<Z(y)=Z^{\prime}(\bar{y})
$$

gelten. Also könnte wegen $Z^{\prime}\left(y^{*}\right)<Z^{\prime}(\bar{y})$ die Näherung $\bar{y}$ keine Lösung des Systems (20) bis (22) sein. Das widerspricht aber der Annahme.

Das transformierte System (20) bis (22) unterscheidet sich vom ursprünglichen Programmierungssystem (1) bis (3) nur durch andere Bezeichnungen. Man wird also versuchen, mit der zugeordneten Näherung als Ausgangsnäherung, nach (13) und (14) weitere verbesserte Näherungen zu berechnen. Sobald das Transformationskriterium wieder erfüllt wird, führt man eine weitere Transformation durch. So fährt man fort, bis man schließlich für irgendein transformiertes System eine Lösung erhalten hat.

Die zugeordnete Lösung für das Ausgangssystem (1) bis (3) ist im System $\mu$, das die Lösung $y_{\text {opt }}^{(\mu)}$ lieferte, direkt enthalten. Denn ist $U_{j}^{(\mu)}-(j=1, \ldots, n)-$ die $j$. Ungleichung dieses Systems, so ist wegen (18)

$$
y_{\mathrm{opt}}=\left(U_{\mathbf{1}}^{(\mu)}\left(y_{\mathrm{opt}}^{(\mu)}\right), \ldots, U_{n}^{(\mu)}\left(y_{\mathrm{opt}}^{(\mu)}\right)\right)
$$

eine Lösung von (1) bis (3).

Bevor der Nachweis erbracht wird, daß der oben beschriebene Weg in endlich vielen Schritten zur Lösung führt, soll im nächsten Abschnitt noch ein Kriterium aufgestellt werden, welches zeigt, wann eine Lösung erreicht ist.

* Denn setzt man in (20) die Abkürzung (23) ein, so folgt $Z^{\prime}(\bar{x})=Z(x)-a_{g} x_{g}+a_{g} U_{g}^{\prime}(\bar{x})$. Nach der Transformationsgleichung (18) ist aber $x_{g}=U_{g}^{\prime}(\bar{x})$ und damit auch $Z^{\prime}(\bar{x})=Z(x)$. 


\subsection{Optimalitätskriterium}

Das folgende Optimalitätskriterium kann zur Entscheidung herangezogen werden, ob sich eine zulässige Näherung durch eindimensionale Verbesserungen und Transformationen noch verbessern läßt, oder eine endliche Lösung bereits erreicht ist.

Optimalitätskriterium. Wenn $y=\left(y_{1}, \ldots, y_{n}\right)$ eine zulässige Näherung von (1) bis (3) ist und

$$
\begin{aligned}
& a_{j}=0 \text { für alle } y_{j}>0 \text { und } \\
& a_{j} \geqq 0 \text { für alle } y_{j}=0
\end{aligned}
$$

gilt, dann ist y sogar eine endliche Lösung des linearen Programmierungssystems.

Ein lineares Programmierungssystem, welches die Bedingung (26) erfüllt, heiße Optimalsystem.

Um die obige Behauptung als Kriterium anwenden zu können, muß folgendes bewiesen werden.

I. Wenn (26) erfüllt wird, ist $y$ eine endliche Lösung.

II. Ist keine eindimensionale Verbesserung mehr möglich, dann erhält man durch Transformation das Optimalsystem.

I kann leicht gezeigt werden. Jede andere zulässige Näherung hat wegen (2) die Form $y^{\prime}=\left(y_{1}^{\prime}, \ldots, y_{n}^{\prime}\right)$ mit $y_{j}^{\prime} \geqq 0$. Nun gilt, wenn man die positiven $a_{j}$ mit $a_{j}^{+}$ und die $a_{j}=0$ mit $a_{j}^{0}$ bezeichnet, die Beziehung

$$
Z\left(y^{\prime}\right)=Z(y)+\sum a_{j}^{+}\left(y_{j}^{\prime}-y_{j}\right)+\sum a_{j}^{0}\left(y_{j}^{\prime}-y_{j}\right) \geqq Z(y),
$$

weil nach Konstruktion $\sum a_{j}^{+}\left(y_{j}^{\prime}-y_{j}\right) \geqq 0$ und $\sum a_{j}^{0}\left(y_{j}^{\prime}-y_{j}\right)=0$ sein muB. Also ist $y$ eine Lösung.

Zum Beweise von II wird angenommen, daß eine endliche Lösung $y$ bereits bekannt sei und für $y$ keine Degeneration herrsche. Dann läßt sich durch Falluntersuchungen zeigen, wie man stets - nach höchstens $n$ Transformationen zu einem Optimalsystem gelangt.

Außer dem Fall, in dem für alle $j$ entweder $a_{j}=0$ und $y_{j}>0$ oder $a_{j} \geqq 0$ und $y_{j}=0$ gilt, d. h. (26) erfüllt wird, existieren noch folgende drei Möglichkeiten.

Für mindestens ein $j$ ist

1. mit $a_{j}>0$ gleichzeitig $y_{j}>0$

2. mit $a_{j}<0$ gleichzeitig $y_{j}>0$

3. mit $a_{j}<0$ gleichzeitig $y_{j}=0$.

In den Fällen 1. bis 3. muß für wenigstens eine Ungleichung $U_{i}^{(3)}$ vom Typ (3) $U_{i}^{(3)}=0$ gelten, weil sonst eine eindimensionale Verbesserung möglich wäre. Solange aber ein $y_{j} \neq 0$ und gleichzeitig ein $U_{i}^{(3)}=0$ ist, kann gemäß (17) eine Transformation durchgeführt werden, welche die Anzahl der $U_{i}^{(3)}=0$ um eins verkleinert. Sollte zuerst kein $y_{j}>0$ mehr existieren, d. h. alle $y_{j}=0$ sein, und noch für eine Ungleichung $U_{i}^{(3)}=0$ gelten, dann würde Degeneration herrschen. Da Degeneration aber ausgeschlossen wurde, sind nach spätestens $n$ Transformationen alle $U_{i}^{(3)} \neq 0$. 
Jetzt muß (26) erfüllt sein, weil sonst entgegen der Voraussetzung eine eindimensionale Verbesserung möglich wäre. Q.e.d.

Bei der Behandlung der Degeneration in Abschnitt 3.1 wird gezeigt, daß das Optimalitätskriterium auch im Falle der hier noch ausgeschlossenen Degeneration anwendbar bleibt. Falls eine Lösung nicht endlich ist, kann man unmittelbar das Ergebnis

$$
Z(y)=-\infty
$$

als Optimalitätskriterium benutzen.

\subsection{Konvergenz}

In diesem Abschnitt soll die Frage untersucht werden, ob man von einer beliebigen Ausgangsnäherung in endlich vielen verbesserten Näherungen zu einer Lösung gelangen kann. Dabei wird im Augenblick die Existenz einer Lösung vorausgesetzt.

Die Fragestellung kann sofort auf zulässige Ausgangsnäherungen eingeschränkt werden. Denn ist die Ausgangsnäherung keine zulässige Näherung, so kann man nach Abschnitt 2.1 durch höchsten $(m+n)$-malige Anwendung der linearen Programmierung zu einer zulässigen Ausgangsnäherung gelangen. Läßt sich also zeigen, daß für eine zulässige Ausgangsnäherung die Anzahl der verbesserten Näherungen, die zu einer Lösung führen, endlich ist, dann ist dasselbe auch gleichzeitig für eine nicht zulässige Ausgangsnäherung bewiesen.

Aus Gründen der Übersichtlichkeit soll der Nachweis für die Konvergenz des Verfahrens in endlich vielen Schritten zuerst für den Fall geführt werden, daß

I. alle Lösungen endlich sind und

II. keine der angenommenen Näherungen mehr als $n$ Ungleichungen exakt erfüllt, d. h. keine Degeneration eintritt.

Unter diesen Voraussetzungen soll also der folgende Satz bewiesen werden.

Satz. Ist y eine zulässige Ausgangsnäherung von (1) bis (3), dann liefert das Verfahren eine endliche Folge

$$
y^{(1)}, y^{(2)}, \ldots, y^{(N)}
$$

von verbesserten Näherungen, für die

$$
Z\left(y^{(1)}\right)>Z\left(y^{(2)}\right)>\cdots>Z\left(y^{(N)}\right)=Z_{\mathrm{opt}}
$$

gilt. Die Anzahl der Transformationen zwischen zwei aufeinander folgenden Näherungen ist ebenfalls endlich.

Die obige Behauptung ist bewiesen, wenn man folgendes zeigen kann:

Lemma A. Zu jeder zulässigen Näherung $y^{(v)}$, für die nicht $Z\left(y^{(v)}\right)=Z_{\text {opt }}$ gilt, liefert das Verfahren nach endlich vielen Transformationen eine verbesserte zulässige Näherung $y^{(v+1)}$ mit

$$
Z\left(y^{(\nu)}\right)>Z\left(y^{(\nu+1)}\right)
$$


Lemma B. Die aus den verbesserten zulässigen Näherungen $y^{(\nu)}$ gebildete Folge der $Z\left(y^{(v)}\right)$ besitzt keinen Häufungspunkt.

Mit Lemma A und B läßt sich die Behauptung leicht beweisen. Denn einmal kann wegen Lemma A die Folge der $Z\left(y^{(v)}\right)$ nicht abbrechen, bevor eine Lösung erreicht ist, und zum anderen ist die Folge der $Z\left(y^{(v)}\right)$ wegen $Z\left(y^{(\nu)}\right) \geqq Z_{\text {opt }}$ beschränkt und muß, da sie nach Lemma B keinen Häufungspunkt besitzt, endlich sein. Es existiert also eine natürliche Zahl $N$, für die $y^{(N)}$ eine Lösung darstellt.

Lemma $A$ soll indirekt bewiesen werden. Es wird also angenommen, daß für eine zulässige Näherung $y$ mit $Z(y)>Z_{\text {opt }}$ keine eindimensionale Verbesserung gefunden werden kann. Dann werden Fallunterscheidungen vorgenommen und in Widerspruch zur Voraussetzung gebracht.

1. $y$ erfüllt keine der Ungleichungen von (2) und (3) exakt. Existiert ein $a_{j} \neq 0$, dann ist nach (13) und (14) unmittelbar eine eindimensionale Verbesserung möglich. Widerspruch zur Voraussetzung.

Daß alle

$a_{j}=0$ sind, ist ebenfalls nicht möglich, weil dann das Optimalitätskriterium erfüllt wäre und $y$ im Widerspruch zur Voraussetzung bereits eine Lösung wäre.

Damit muß $y$ wenigstens eine der Ungleichungen von Typ (2) oder (3) exakt erfüllen.

2. $y$ erfüllt nur Ungleichungen vom Typ (2) exakt. Für

$a_{j}<0$ ist nach (13) und (14) ohne weitere Transformation eine eindimensionale Verbesserung möglich, im Widerspruch zur Voraussetzung.

Wären aber alle

$a_{j} \geqq 0$ und $y=y^{0}=(0, \ldots, 0)$, dann wäre das Optimalitätskriterium erfüllt. Das gleiche gilt auch für $y \neq y^{0}$, sobald für jedes $y_{j} \neq 0$ stets $a_{j}=0$ ist. Sollte aber für ein $y_{j} \neq 0$ auch $a_{j}>0$ sein, dann wäre wieder unmittelbar eine eindimensionale Verbesserung möglich.

Damit sind alle möglichen Fälle von 2 zum Widerspruch geführt worden. Es bleibt nur noch der Fall zu betrachten, daß

3. y genau $n-t(0 \leqq t<n)$ Ungleichungen vom Typ (3) exakt erfüllt. In diesem Falle sind - Degeneration ausgeschlossen - wenigstens $n-t$ der $y_{j}>0$. Jede jetzt durchgeführte Transformation erniedrigt die Anzahl der von $y$ exakt erfüllten Ungleichungen vom Typ (3) um eins. Nach $n-t$ Transformationen wird dann ein lineares Programmierungssystem erhalten, in dem keine Typ-(3)-Ungleichung mehr von $y$ exakt erfüllt ist. Damit läßt sich Fall 3 auf Fall 2 zurückführen und in Widerspruch zur Voraussetzung bringen.

Es existiert also keine zulässige Näherung, ausgenommen eine Lösung, zu der es keine eindimensionale Verbesserung gibt. Das ist aber die Behauptung von Lemma A.

Auch der Beweis von Lemma B wird indirekt geführt. Es wird daher angenommen, das Programmierungsverfahren liefere eine unendliche Folge $y^{(v)}$ von verbesserten Näherungen, für welche die Folge $\left\{Z\left(y^{(\nu)}\right)\right\}$ gegen einen Wert $A=Z\left(y^{*}\right)$ konvergiere. Dabei sei $A$ der größte aller Häufungspunkte.

Z. Wahrscheinlichkeitstheorie, Bd. 1 
Als erstes wird gezeigt, wie zu jedem vorgegebenen $\varepsilon>0$ ein $\delta>0$ existiert, so daß von einem bestimmten $v$ ab mit

auch stets der Abstand

$$
\left|Z\left(y^{(p)}\right)-Z\left(y^{*}\right)\right|<\delta
$$

wird.

$$
\left|y^{(\nu)}-y^{*}\right|<\varepsilon(\delta)
$$

Dies ist leicht zu zeigen. Abgesehen vom trivialen Fall, in dem alle $a_{j}=0$ sind, existiert wegen der endlichen Anzahl von möglichen, verschiedenen Transformationen immer ein absolut kleinster Koeffizient $a_{f} \neq 0$. Weil $Z\left(y^{(v)}\right)$ gegen $A$ konvergiert, muß von einem $v$ ab die Relation

gelten. Aus

$$
\left|Z\left(y^{(v)}\right)-Z\left(y^{(\nu+1)}\right)\right|=|\Delta Z|==\left|a_{j} \Delta y_{j}\right|<\delta
$$

folgt weiter

$$
\left|a_{f} \Delta y_{j}\right| \leqq\left|a_{j} \Delta y_{j}\right|<\delta
$$

$$
\left|\Delta y_{j}\right|<\frac{\delta}{\left|a_{f}\right|}=\varepsilon_{1}(\delta)
$$

Wegen $y^{(v+1)}=y^{(v)}+\Delta y_{j}^{\star}$ gilt dann auch

$$
\left|\Delta y_{j}\right|=\left|y^{(\nu+1)}-y^{(\nu)}\right|<\varepsilon_{1} .
$$

Damit ist gezeigt, daß die Folge $y^{(v)}$ wie behauptet gegen einen Punkt konvergiert, der wegen der vorausgesetzten Konvergenz der Folge der $Z\left(y^{(\nu)}\right)$ gegen $Z\left(y^{*}\right)$ nur $y^{*}$ sein kann.

Bezeichnet man nun die von $y^{*}$ exakt bzw. nicht exakt erfüllten Ungleichungen mit $U_{i *}$ bzw. $\bar{U}_{i}$, dann läßt sich $\delta>0$ so wählen, daß von einem bestimmten $v$ ab mit

auch stets

$$
\left|Z\left(y^{(v)}\right)-Z\left(y^{*}\right)\right|<\delta
$$

$$
U_{i *}\left(y^{(p)}\right)<\bar{U}_{i}\left(y^{(p)}\right)
$$

gilt.

Die erste zulässige Näherung $y^{(v)}$, welche dieser Bedingung entspricht, kann nach Voraussetzung in endlich vielen Iterationsschritten erreicht werden. Nun läßt sich wie folgt zeigen, daß man unter der Voraussetzung (28) auch nach endlich vielen Schritten zu $y^{*}$ gelangen kann: Wegen (28) werden die verbesserten Näherungen $y^{(\nu+\mu)}(\mu>0)$ nur die Ungleichungen $U_{i *}$ exakt erfüllen. Es können also nur die Nebenbedingungen $U_{i *}$ als Transformationsgleichungen herangezogen werden und damit die Form des Types (2) annehmen (vgl. Abschnitt 2.3). Gehört aber die Ungleichung $U_{i *}$ erst einmal zu den Typ-(2)-Ungleichungen, dann bleibt sie es wegen (17) und (28) auch. Weil jedoch nach (15) auf mindestens $2 m+n+1$ eindimensionale Verbesserungen eine Transformation folgt, so haben nach spätestens $n$ Transformationen und $n(2 m+n+1)$ eindimensionalen Verbesserungen

\footnotetext{
* Symbolische Schreibweise für Relation (10).
} 
alle Ungleichungen $U_{i *}$ die Form von (2). Sollte in diesem Zustand für die entsprechende verbesserte Näherung $y^{\prime}$ noch $Z\left(y^{\prime}\right)>Z\left(y^{*}\right)$ gelten, so wird man $y^{*}$ ohne weitere Transformationen nach höchstens $n$ eindimensionalen Verbesserungen mit $\left|\Delta y_{j}\right|=\left|y_{j}^{\prime}-y^{*}\right|$ erreichen.

Damit ist im Widerspruch zur Annahme gezeigt, daß man mit Hilfe des Programmierungsverfahrens in endlich vielen Iterationsschritten die zulässige Näherung $y^{*}$ erhält. Die Folge der $Z\left(y^{(v)}\right)$ kann daher keinen Häufungspunkt besitzen und muß wegen Lemma A nach endlich vielen Schritten mit $Z\left(y^{(N)}\right)=Z_{\text {opt }}$ abbrechen.

Also gelangt man, Degeneration ausgeschlossen, von jeder Ausgangsnäherung nach endlich vielen Iterationsschritten zu einer endlichen Lösung. Dasselbe wird später auch für die hier noch ausgeschlossenen. Sonderfälle der Degeneration und einer unendlichen Lösung bewiesen werden.

\section{Sonderfälle}

Dieses Kapitel soll den Sonderfällen gewidmet sein, die bei der Lösung linearer Programmierungssysteme auftreten können.

Im Normalfalle hat das Programmierungssystem bereits die Form (1) bis (3), man kennt eine zulässige Ausgangsnäherung und gelangt ohne Degeneration von dieser nach endlich vielen verbesserten Näherungen zur einzigen endlichen Lösung. Für den Normalfall ist die Beschreibung des Lösungsverfahrens in Kapitel 2 ausreichend. Es sind aber bei den Beweisen und Lösungsanweisungen noch verschiedene Fragen ungeklärt geblieben, die alle bei der Behandlung der Sonderfälle ihre Lösung finden werden.

\subsection{Degeneration}

Zu den wichtigsten Sonderfällen gehört die Degeneration. Wie schon erwähnt, sagt man, für eine Näherung herrsche Degeneration, wenn die Näherung mehr als $n$ der Ungleichungen von (2) und (3) exakt erfüllt.

Als Folge der Degeneration wurden bei der Herleitung des Lösungsverfahrens in Kapitel 2 einige Fragen offen gelassen und bedürfen noch der Klärung. Sie lauten:

I. Wie ist weiter vorzugehen, wenn bei Degeneration nach (17) $y_{g}=0$ wird?

II. Gilt das Optimalitätskriterium bei Degeneration?

III. Gelangt man auch im Falle der Degeneration nach endlich vielen Schritten zu einer Lösung?

Alle drei Fragen lassen sich gemeinsam beantworten. Weil - wie sich zeigen wird - I mit der Lösung von II beantwortet ist und III sich als einfache Folgerung aus II ergibt, ist es zweckmäßig, gleich mit dem Beweis für die Gültigkeit des Optimalitätskriteriums im Falle der Degeneration zu beginnen.

Nach 2.4 kann man dazu von einem Programmierungssystem mit endlicher Lösung ausgehen, das die folgenden Eigenschaften besitzt. 
A. Die Lösung $y^{0}=(0, \ldots, 0)$ erfüllt außer allen Ungleichungen vom Typ (2) noch $s$ Ungleichungen $U_{i^{*}}^{(3)}$ vom Typ (3) exakt, d. h., es herrsche Degeneration vom Grade $s$.

B. Es existiert mindestens ein $a_{j}<0$ und zu jedem dieser $a_{j}^{-}$gibt es mindestens ein $b_{i^{*}, j}<0$. Das heißt, es ist keine eindimensionale Verbesserung möglich.

Das Vorgehen entspricht den folgenden Gedanken. Man beseitigt die Degeneration, in dem man zu allen Ungleichungen $U_{i^{*}}^{(3)}$ eine beliebig kleine Größe $\varepsilon>0$ addiert und nach den für den Normalfall geltenden Regeln fortfährt. Dabei stellt sich heraus, daß die Auswahl der Transformationsvariablen $x_{g}$ und der Transformationsgleichung $U_{n+k}$ unabhängig von der Größe $\varepsilon$ ist. Das heißt, setzt man $\varepsilon=0$ und wendet ohne eindimensionale Verbesserung die so gewonnenen Regeln für die Transformation an, dann erhält man dieselben transformierten Systeme wie bei $\varepsilon \neq 0$. Letztere führen aber in endlich vielen. Schritten zum Optimalsystem.

Die detaillierte Darstellung lautet folgendermaßen.

1. Addiere zu jeder von $y^{0}$ exakt erfüllten Ungleichung $U_{i *}$ die Größe $\varepsilon>0$. Dabei ist $\varepsilon$ so klein gewählt, daß - solange für das Programmierungssystem $B$ gilt - eine eindimensionale Verbesserung nur die erweiterten Ungleichungen $U_{i *}+\varepsilon$ exakt erfüllen kann.

2. Jetzt existiert stets eine eindimensionale Verbesserung, für die wegen $U_{i *}\left(y^{0}\right)=0$ nach (13) und (14)

$$
\Delta Z=\min _{j} a_{j} \Delta y_{j}
$$

mit

$$
\Delta y_{j}= \begin{cases}\varepsilon \max _{i^{*}}\left(-\frac{1}{b_{i *, j}^{+}}\right) & \text {für } \quad a_{j}>0 \\ \varepsilon \min _{i^{*}}\left(-\frac{1}{b_{i *, j}^{-}}\right) & \text {für } \quad a_{j}<0\end{cases}
$$

gilt.

3. Für die verbesserte Näherung $y^{\prime}$, die eine der Ungleichungen $U_{i_{*}}$, z. B. $U_{n+k}$, exakt erfüllt, ist genau ein $y_{j}^{\prime} \neq 0$. Es ist also eine Transformation nach (17) möglich. Nennt man die Transformationsvariable wieder $x_{g}$ und die Transformationsgleichung $U_{n+k}$, so läßt sich $g$ und $n+k$ auch direkt aus (13D) und (14D) bestimmen. Denn es gilt

$$
\Delta Z=\min _{j} a_{j} \Delta y_{j}=\varepsilon \min _{j} \max _{i^{*}}\left(-\frac{a_{j}^{+}}{b_{i *, j}^{+}}, \quad-\frac{a_{\bar{i}}^{-}}{b_{i *, j}^{+}}\right)=-\frac{a_{g}}{b_{n+k, g}} \varepsilon .
$$

Das heißt, man kann $g$ und $n+k$ unabhängig von $\varepsilon$ so wählen, daß

$$
\frac{a_{g}}{b_{n+k, g}}=\max _{j} \min _{i^{*}}\left(\frac{a_{j}^{+}}{b_{i *, j}^{+}}, \frac{a_{\bar{j}}^{-}}{b_{i *, j}^{-}}\right)
$$

gilt. Falls es mehrere Paare $\left(i^{*}, j\right)$ gibt, welche $(17 \mathrm{D})$ erfüllen, dann kann man ein beliebiges Paar davon als $(n+k, g)$ auswählen. 
4. Führt man die Transformation nach (17D) durch, dann hat die zu $y^{\prime}$ zugeordnete Näherung $y^{\prime 0}$ wieder die Form von $y^{0}$. Für diese Näherung ist die Degeneration aber um mindestens einen Grad kleiner.

5a. Tritt für $y^{\prime}{ }^{0}$ noch Degeneration auf, dann wird nach 1. fortgefahren. Nach spätestens $s$ Transformationen gelangt man so zum Fall $5 \mathrm{~b}$.

$5 \mathrm{~b}$. Ist für $y^{\prime 0}$ keine Degeneration vorhanden und noch mindestens ein $a_{j}<0$ (sonst wäre das Optimalitätskriterium bereits erfüllt), dann kann man mit 2 . fortfahren.

6. Für den Normalfall, wie er in 5 b. auftritt*, wird nach endlich vielen Schritten das Optimalitätskriterium erfüllt.

7. Da nun die Vorschrift für die Auswahl der Transformationsindizes von $\varepsilon$ unabhängig ist, kann man $\varepsilon=0$ setzen und gelangt so - ohne eindimensionale Verbesserung - in endlich vielen, nach (17D) durchgeführten Transformationen. zum Optimalsystem.

Damit ist die Gültigkeit des Optimalitätskriteriums für endliche Lösungen auch bei Degeneration bewiesen.

Wenn Degeneration auftritt, dann ist es zweckmäßig, auch schon für $y_{g *} \neq \mathbf{0}$ die Relation (17D) zur Auswahl der Transformationsgleichung heranzuziehen. Das heißt, so bald ein Paar $\left(i^{*}, g^{*}\right)$ existiert, für welches der Quotient $a_{g *} / b_{i *, g *}$ eine positive Zahl ist, kann man $g$ und $n+k$ aus (17D) bestimmen. Falls mehrere Paare $\left(i^{*}, g^{*}\right)$ Relation (17D) erfïllen, wird ein beliebiges Paar davon als $(n+k, g)$ ausgewählt.

Ist der Quotient aber für alle Paare $\left(i *, g^{*}\right)$ kleiner oder gleich null, dann besteht für die Transformationsgleichung nur die Bedingung $b_{n+k, g} \neq 0$ und $g$ und $n+k$ werden dann nach Abschnitt 2.3 bestimmt. Damit ist Frage I geklärt.

Auch Frage III kann jetzt positiv beantwortet werden. Tritt im Laufe der Rechnung für eine Näherung Degeneration auf, so muß bei Anwendung von (17D) nach endlich vielen Transformationen entweder eine eindimensionale Verbesserung möglich sein, oder eine Lösung erreicht werden. Da es wegen der $n+m$ Ungleichungen nur eine endliche Anzahl von Näherungen gibt, die $A$ und $B$ erfüllen, erreicht man nach endlich vielen Schritten eine endliche Lösung *ᄎ.

\subsection{Unendliche Lösung}

Gibt es für jede beliebig große, positive Zahl $G$ stets eine zulässige Näherung $y_{G}$, für die $Z\left(y_{G}\right)<-G$ gilt, dann sagt man, das Programmierungssystem (1) bis (3) besitzt eine unendliche Lösung. Diese tritt stets dann auf, wenn bei der eindimensionalen Verbesserung für ein $\alpha_{j}<0$ in (14) $\triangle y=\infty$ und damit $\triangle Z=-\infty$ wird. Es müssen dann also

$$
\text { alle zu einem } a_{j}<0 \text { gehörigen } b_{i j} \geqq 0 \text { sein, }
$$

* Weil die Vorschrift, daß nach spätestens $2 m+n+1$ eindimensionalen Verbesserungen eine Transformation folgen muß, willkürlich gewählt wurde, kann man auch $2 m+n+1$ durch 1 ersetzen, ohne die Beweise wesentlich ändern zu müssen.

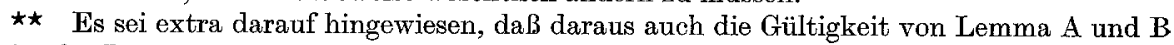
in 2.5 für Degeneration folgt. 
so daß in (12) die Menge der $-U_{i}(y) / b_{i j}^{-}$die leere Menge $L$ ist*

Im folgenden soll nun das im Kapitel 2 entwickelte Verfahren so ergänzt werden, daß auch für ein Programmierungssystem mit unendlicher Lösung diese nach endlich vielen Schritten erreicht wird.

Es sei $y^{(\mu) 0}=(0, \ldots, 0)$ eine zulässige Näherung des durch Transformationen aus (1) bis (3) erhaltenen Systems $\mu$. Dann existiert eine negative Zahl ${ }^{\star \star} M$, so daß für jedes der endlich vielen $\mu$

$$
M<Z^{(\mu)}\left(y^{(\mu) 0}\right)
$$

gilt. Gibt man ein solches $M$ vor, so wird als Folge von Lemma A und B aus 2.5 nach endlich vielen Schritten eine verbesserte Näherung $y^{\prime}$ erreicht, für die

$$
Z^{\prime}\left(y^{\prime}\right)<M
$$

ist.

Wenn man jetzt von einer Näherung $y^{\prime}$ an, die (31) erfüllt, bei der Berechnung der eindimensionalen Verbesserung nach (13) und (14) nur solche $j$ berücksichtigt, für die

$$
y_{j} \neq 0
$$

gilt, dann gelangt man nach endlich vielen Schritten zu einer unendlichen Lösung.

Das ist leicht zu zeigen. Angenommen eine unendliche Lösung werde unter diesen Bedingungen nicht in endlich vielen Schritten erreicht. Dann erhält man wegen (17) nach spätestens $n$ Transformationen ein System, für welches die zuletzt erhaltene verbesserte Näherung die Form $y^{(\mu) 0}$ hat.

Für $y^{(\mu) 0}$ gilt aber

$$
Z^{(\mu)}\left(y^{(\mu) 0}\right) \leqq Z\left(y^{\prime}\right)<M,
$$

was ein Widerspruch zu (30) ist, q.e.d.

Damit ist gezeigt, daß man auch unendliche Lösungen in einer endlichen Anzahl von Iterationsschritten erreichen kann.

Für die numerische Berechnung beachte man den folgenden Hinweis. Es dürfte sehr umständlich sein, den Wert von $M$ gleich so zu bestimmen, daß (30) mit Sicherheit erfüllt ist. Daher wird man zuerst einen Näherungswert $M_{0}$ (z. B. $M_{0}=-10^{6}$ ) annehmen, aus dem man nötigenfalls nach der willkürlich angenommenen Beziehung

$$
M_{y}=M_{0} \cdot 10^{2 v} \quad(v=0,1,2, \ldots)
$$

bessere Näherungswerte $M_{\nu}$ berechnet.

Hat man nun einen Wert $M_{\nu}$ gewählt, und tritt nach endlich vielen Transformationen die verbesserte Näherung $y^{(\mu) 0}$ auf, dann gilt offensichtlich noch

* Geometrisch bedeutet dies, daß der durch (2) und (3) definierte Bereich unbeschränkt ist.

$\star \star \quad$ Die Existenz von $M$ ist gesichert, weil $Z^{(\mu)}\left(y^{(\mu) 0}\right)=a_{0}^{(\mu)}$ ist und wegen (23) mit $a_{0}$ auch $a_{0}^{(\mu)}$ einen endlichen Wert annimmt. 
$M_{v}>M$. Jetzt ersetzt man $M_{\nu}$ durch $M_{\nu+1}$ und berechnet die eindimensionale Verbesserung so lange wieder für alle $j$, bis (31) von einer verbesserten Näherung erfüllt wird. Es ist klar, daß man so nach endlich vielen Wiederholungen zu einem Wert $\nu=\nu^{*}$ kommt, für den $M_{\nu *} \leqq M<Z^{(\mu)}\left(y^{(\mu) 0}\right)$ gilt. Wie bereits bewiesen, erhält man aber für diesen Fall eine unendliche Lösung nach endlich vielen verbesserten Näherungen.

\subsection{Mehrfache Lösung}

Man sagt, das lineare Programmierungssystem (1) bis (3) besitze eine mehrfache Lösung, wenn es zwei verschiedene zulässige Näherungen $y^{(1)}$ und $y^{(2)}$ gibt, für die

$$
Z\left(y^{(1)}\right)=Z\left(y^{(2)}\right)=Z_{\mathrm{opt}}
$$

gilt. Nach Abschnitt 2.4 und 2.5 ist auch für diesen Sonderfall das Optimalitätskriterium gültig und das Erreichen einer Lösung in endlich vielen Schritten ge. sichert.

Um beurteilen zu können, wann eine mehrfache Lösung vorliegt, sollen die Bedingungen für ihr Auftreten zusammengestellt werden. Das wird wieder an Hand von Fallunterscheidungen erfolgen.

Als erstes kann vorausgesetzt werden, daß das Optimalitätskriterium und für mindestens ein $j=j^{*}$ die notwendige Voraussetzung $a_{j *}=0$ erfüllt ist. Wenn man die bekannte Lösung mit $y^{\prime}$ und die Ungleichungen des Optimalsystems mit $U^{\prime}$ bezeichnet, dann bleiben die folgenden Fälle zu untersuchen.

1. Für alle Ungleichungen $U_{i}^{\prime(3)}$ vom Typ (3) gilt

$$
U_{i}^{\prime(3)}\left(y^{\prime}\right)>0 \text {. }
$$

2. Es existiert mindestens ein $i=i *$ mit

$$
U_{i^{*}}^{\prime(3)}\left(y^{\prime}\right)=0 \text {. }
$$

a) Dabei ist ein $y_{j^{*}}^{\prime}>0$ und für dieses $j^{*}$

风) alle $b_{i *, j *}=0$,

B) ein $b_{i *, j *} \neq 0$.

b) Hier sind alle $y_{j *}^{\prime}=0$ und für ein $j^{*}$ alle $b_{i *, j *} \geqq 0$.

c) Es seien alle $y_{j *}^{\prime}=0$ und für jedes $j^{*}$ existiere ein

$$
b_{i *, j *}<0 \text {. }
$$

Nun folgt unmittelbar aus (12) und der Existenz von $a_{j *}=0$, daß es in den Fällen 1, $2 \mathrm{a} \alpha$ und $2 \mathrm{~b}$ eine mehrfache Lösung gibt. So lange der Fall $2 \mathrm{a} \beta$ gilt, kann man nach Abschnitt 2.3 Transformationen durchführen, bis $2 \mathrm{a} \beta$ auf einen der anderen Fälle zurückgeführt worden ist. Damit bleibt nur noch $2 \mathrm{e}$ zu klären.

In diesem Falle existiert offensichtlich eine mehrfache Lösung, wenn man durch ausschließliche Änderung der $y_{j^{*}}^{\prime}$ eine zulässige Näherung $y$ finden kann, die verschieden von $y^{0}=(0, \ldots, 0)$ ist. 
Man betrachtet also alle von $y^{\prime}$ exakt erfüllten Ungleichungen $U_{i^{*}}^{\prime}\left(y^{\prime}\right)=0$ und setzt in diesen alle $b_{i *, j}=0$, für die $a_{j}>0$ gilt. Die so erhaltenen Ungleichungen kann man in der Form

und

$$
\begin{aligned}
& u_{j * 1}\left(x^{*}\right)=x_{j * 1} \quad \geqq 0 \\
& u_{j^{*} e}\left(x^{*}\right)=\quad x_{j^{*} e} \geqq 0
\end{aligned}
$$

$$
\begin{aligned}
& u_{i^{*} 1}\left(x^{*}\right)=b_{i^{*} 1, j^{* 1}} x_{j^{* 1}}+\cdots+b_{i^{* 1}, j^{*} e} x_{j^{*} e} \geqq 0 \\
& \ldots \ldots \ldots \\
& u_{i^{*} c}\left(x^{*}\right)=b_{i^{*} c, j^{* 1}} x_{j^{* 1}}+\cdots+b_{i^{*_{c}, j^{*} e}} x_{j^{*} e} \geqq 0
\end{aligned}
$$

zusammenfassen. Dabei wurden die Bezeichnungen $x^{*}=\left(x_{j^{* 1}}, \ldots, x_{j^{*}}\right)$ und $i_{v}^{*}=j_{e+v}^{*}$ benutzt.

Nun führt man eine Hilfsfunktion

$$
z\left(x^{*}\right)=\vec{a}_{j *_{1}} x_{j{ }_{1}}+\cdots+\bar{a}_{j_{e},} x_{j^{*}}
$$

mit $\bar{a}_{j^{*} 1}=\cdots=\bar{a}_{j^{*} e}=-1$ ein und minimiert $(1 \mathrm{M})$ unter den Nebenbedingungen (2M) und (3M). Wenn $z_{\text {opt }}<0$ ist, dann und nur dann existiert auch im Fall $2 \mathrm{c}$ eine mehrfache Lösung. Es ist klar, daß man das Verfahren abbricht, sobald man. eine Näherung $y^{*}$ gefunden hat, für die

$$
z\left(y^{*}\right)<0
$$

gilt. Denn mit (34) ist die Existenz einer mehrfachen Lösung für den Fall 2c bereits sichergestellt. Damit ist gezeigt, wie man in jedem Falle entscheiden kann, ob eine mehrfache Lösung existiert.

Leider ist eine Darstellung der Gesamtheit der Lösungen für das System (1) bis (3) in expliziter Form nur in Ausnahmefällen möglich. Diese Schwierigkeit läßt sich jedoch bei praktischen Anwendungen oft durch eine zweite, bedingte Programmierung umgehen. Denn herrscht unter den Lösungen eine Preferenz $P$ - sonst bestünde ja kein Bedürfnis, alle Lösungen zu kennen -, so wird man versuchen, diesen Zusammenhang in einer linearen Gleichung der Form

$$
P(x)=p_{1} x_{1}+\cdots+p_{n} x_{n}+p_{0}
$$

$\mathrm{zu}$ erfassen.

Ist dies möglich, so kann man (35) optimieren unter den Nebenbedingungen, daß jede Lösung $Y=\left(Y_{1}, \ldots, Y_{n}\right)$ von (35) die Gleichung

$$
a_{1} x_{1}+\cdots+a_{n} x_{n}+a_{0}-Z_{\mathrm{opt}}=0
$$

und (2) und (3) erfüllt. Wie dieses System auf die Normalform gebracht werden kann, wird in Abschnitt 3.5 beschrieben.

Wie bereits erwähnt, handelt es sich hier um eine bedingte Programmierung, weil sie unter der Voraussetzung durchgeführt wird, daß die erste Zielfunktion $Z$ mit Vorrang zum Minimum gemacht wurde. Aus dem oben Dargestellten ergibt 
sich sofort, daß eine bedingte, nach einem ganz anderen Gesichtspunkt erstellte Programmierung nur dann sinnvoll ist, wenn die erste Programmierung eine mehrfache Lösung besitzt.

\subsection{Existenz einer Lösung}

Eine zulässige Näherung $y$ des linearen Programmierungssystems (1) bis (3) nennt man eine Lösung, wenn entweder

$$
Z(y)=-\infty
$$

oder für alle anderen zulässigen Näherungen $\bar{y}$

$$
Z(\bar{y}) \geqq Z(y)
$$

gilt. Der Wert $Z(y)$ wird dann mit $Z_{\text {opt }}$ bezeichnet.

Wann existiert nun für das lineare Programmierungssystem (1) bis (3) eine Lösung?

Aus der oben genannten Definition folgt, daß das Vorhandensein einer zulässigen Näherung eine notwendige Voraussetzung für die Existenz einer Lösung ist. Diese Voraussetzung ist aber, wie unten bewiesen wird, auch hinreichend.

Setzt man die Existenz einer zulässigen Näherung voraus, dann gibt es nach Abschnitt 3.2 stets eine Zahl $M$, für die (30) gilt. Jetzt sind zwei Fälle möglich:

1. Für alle zulässigen Näherungen gilt

$$
Z(y) \geqq M \text {. }
$$

2. Es existiert eine zulässige Näherung $y=y_{M}$ mit

$$
Z\left(y_{M}\right)<M \text {. }
$$

Im ersten Falle liefert nun das Verfahren von einer beliebigen zulässigen Näherung ausgehend eine Folge $y^{(v)}$, für welche die abnehmende Folge der $Z\left(y^{(v)}\right)$ nach Lemma B keinen Häufungspunkt besitzt. Weil nach Voraussetzung $Z\left(y^{(p)}\right) \geqq M$ ist, bricht diese Folge mit einem Wert $Z\left(y^{(N)}\right)$ ab. Nach Lemma A liefert das Verfahren aber nur dann keine verbesserte Näherung mehr, wenn für alle anderen zulässigen Näherungen $\bar{y}$ die Ungleichung $Z(\bar{y}) \geqq Z\left(y^{(N)}\right)=Z_{\text {opt }}$ gilt. Damit ist $y^{(N)}$ nach Definition eine endliche Lösung.

Im zweiten Falle benutzt man direkt die Ergebnisse des Abschnittes 3.2. Würde keine unendliche Lösung existieren, so müßte man nach spätestens $n$ Transformationen von $y_{M} z u$ einer Näherung $y^{(\mu) 0}$ kommen, für die im Widerspruch zur Definition von $M$

gilt. Q.e.d.

$$
Z^{(\mu)}\left(y^{(\mu) 0}\right)<M
$$

Damit ist also gezeigt, daß das lineare Programmierungssystem (1) bis (3) dann und nur dann eine Lösung besitzt, wenn eine zulässige Näherung existiert*.

\footnotetext{
* Kriterien, wann ein lineares Ungleichungssystem eine Lösung besitzt, wurden bereits von Motzk TN [10] und KuHo-Tuoker [8] aufgestellt. Leider sind diese Kriterien für die Anwendungen nicht sehr geeignet.
} 
Nun wurde in Abschnitt 2.1 gezeigt, wie man mit Hilfe der linearen Programmierung eine zulässige Näherung für (1) bis (3) berechnen kann, falls diese überhaupt existiert. Dabei wurde auch erwähnt, daß immer dann keine zulässige Näherung und folglich auch keine Lösung existiert, wenn keine Lösung * des Systems. (7) und (5) eine der Ungleichungen (6) verletzt. Es wird also spätestens bei der Berechnung einer zulässigen Näherung für das System (1) bis (3) entschieden, ob eine Lösung existiert oder nicht.

\subsection{Normalform}

Für die Erläuterung des Verfahrens in Kapitel 2 wurde angenommen, daß das lineare Programmierungssystem bereits in der Normalform (1) bis (3) gegeben sei. Beim Aufstellen von linearen Programmierungsmodellen erhält man aber oft anders geartete Systeme. Wie diese auf die Normalform gebracht werden können, soll im folgenden an Hand der einzelnen Abweichungen erläutert werden.

I. Maximierung.

Jede Maximierung kann auf eine Minimierung zurückgeführt werden. Denn lautet die Zielfunktion etwa: ,Maximiere

$$
W=a_{1} x_{1}+\cdots+a_{n} x_{n}+a_{0}{ }^{\prime \prime}
$$

und multipliziert man beide Seiten dieser Gleichung mit - 1, dann erhält man die äquivalente Zielsetzung: „Minimiere

$$
Z=-W=-a_{1} x_{1}-\cdots-a_{n} x_{n}-a_{0} . "
$$

Ist $y=\left(y_{1}, \ldots, y_{n}\right)$ mit $Z(y)=C$ eine Lösung der Minimumaufgabe, dann erhält man für die entsprechende Lösung der Maximumaufgabe

$$
y=\left(y_{1}, \ldots, y_{n}\right) \text { mit } W(y)=-Z(y)=-C .
$$

III. $\bar{U}_{i}(x) \leqq 0$.

a) Jede Ungleichung vom Typ (3)

$$
\bar{U}_{i}(x)=b_{i 1} x_{1}+\cdots+b_{i n} x_{n}+b_{i 0} \leqq 0
$$

kann man durch Multiplikation mit -1 auf die gewünschte Form

bringen.

$$
U_{i}(x)=-\bar{U}_{i}(x)=-b_{i 1} x_{1}-\cdots-b_{i n} x_{n}-b_{i 0} \geqq 0
$$

b) Ist $\bar{U}_{j}(x) \leqq 0$ eine Ungleichung vom Typ (2), gilt also

$$
\bar{U}_{j}(x)=\bar{x}_{j} \leqq 0,
$$

dann erhält man nach Multiplikation mit -1

$$
U_{j}(x)=-\bar{U}_{j}(x)=-\bar{x}_{j} \geqq 0 .
$$

Ersetzt man nun im ganzen Programmierungssystem $-\bar{x}_{j}$ durch $x_{j}$, so hat man ebenfalls die Normalform erhalten.

* Als Folge der Konstruktion existiert für das System (7) und (5) immer eine zulässige Näherung und damit nach dem oben gesagten auch stets eine Lösung. 
Wenn $\bar{y}=\left(y_{1}, \ldots, y_{j}, \ldots, y_{n}\right)$ eine Lösung der Normalform ist, dann stellt

$$
y=\left(y_{1}, \ldots,-y_{j}, \ldots, y_{n}\right)
$$

die entsprechende Lösung der Ausgangsform des Falles II, b dar.

III. $U_{i}(x)=0$.

a) Ist $U_{j}(x)=x_{j}=0$ eine der Relationen vom Typ (2), dann setzt man im ganzen Programmierungssystem $x_{j}=0$ und löst das um die Variable $x_{j}$ und die Ungleichung $U_{j}(x)=x_{j}=0$ reduzierte System.

Wenn $\bar{y}=\left(y_{1}, \ldots, y_{j-1}, y_{j+1}, \ldots y_{n}\right)$ eine Lösung des reduzierten Systems ist, so ergibt

$$
y=\left(y_{1}, \ldots, y_{j-1}, y_{j}=0, y_{j+1}, \ldots, y_{n}\right)
$$

eine Lösung des ursprünglichen Systems.

b) Gilt in einer Ungleichung des Typs (3) das Gleichheitszeichen:

$$
U_{i}(x)=b_{i 1} x_{1}+\cdots+b_{i n} x_{n}+b_{i 0}=0,
$$

dann löst man diese Gleichung nach einer Variablen auf, z. B. falls $b_{i n} \neq 0$ ist, nach $x_{n}$. Darauf setzt man

$$
U_{n}^{\prime}(\bar{x})=x_{n}=-\frac{1}{b_{i n}}\left(b_{i 1} x_{1}+\cdots+b_{i, n-1} x_{n-1}+b_{i 0}\right)
$$

in das Programmierungssystem ein und bringt dieses durch die Reduktion um die Variable $x_{n}$ und die Nebenbedingung $U_{i}(x)$ auf die Normalform. Ist $\vec{y}=\left(y_{1}\right.$, $\ldots, y_{n-1}$ ) eine Lösung des reduzierten Systems, so wird die entsprechende Lösung des ursprünglichen Systems durch

gegeben.

$$
y=\left(y_{1}, \ldots, y_{n-1}, y_{n}=U_{n}^{\prime}(\bar{y})\right)
$$

IV. $U_{i}(x)>0$ oder $U_{i}(x)<0$.

Weil dieser Fall bei den praktischen Anwendungen der linearen Programmierung von geringer Bedeutung ist, wurde er nicht im Verfahren berücksichtigt. Ein derartiger Versuch würde auch bezüglich der Definition einer Lösung einige Schwierigkeiten bereiten, denn im Sinne der Definition (38) braucht trotz des Vorhandenseins einer zulässigen Näherung keine Lösung zu existieren.

Nun könnte man allerdings die Definition einer Lösung erweitern, indem man alle echten Ungleichungen $U_{i}(x)$ durch Subtraktion bzw. Addition von $\varepsilon>0 \mathrm{zu}$

$$
\bar{U}_{i}(x)=U_{i}(x)-\varepsilon \geqq 0 \quad \text { bzw. } \quad \bar{U}_{i}(x)=U_{i}(x)+\varepsilon \leqq 0
$$

ergänzt, und wenn $y_{\text {opt }}(\varepsilon)$ eine Lösung des ergänzten Systems ist,

$$
Y_{\mathrm{opt}}=\lim _{\varepsilon=0} y_{\mathrm{opt}}(\varepsilon)
$$

als Lösung des ursprünglichen Systems definieren. Dann hätte man aber in Kauf zu nehmen, daß $Y_{\text {opt }}$ eventuell keine zulässige Näherung darstellt, sondern gewisse Nebenbedingungen geringfügig verletzt. Sollte in der Praxis jemals eine echte Ungleichung als Restriktion auftreten, so könnte man in diesem Sinne vorgehen.

Damit sind die wichtigsten bei den Anwendungen auftretenden Fälle auf die Normalform zurückgeführt. 


\section{Literatur}

[1] ARrow, K. J., L. Horwicz and H. Uzawa (editors): Studies in Linear and Non-Linear Programming. Stanford University Press, Stanford, Cal., 1958.

[2] Brate, E. M. L.: An Alternative Method of Linear Programming. Proc. Cambridge philos. Soc. 50, 513-523 (1954).

[3] Chaneres, A.: Optimality and Degeneracy in Linear Programming. Econometrica 20, $160-170$ (1952).

[4] Dantzig, G. B., A. Orden and P. Wolfe: The Generalized Simplex Method for Minimizing a Linear Form under Linear Inequality Constraints. Pacific J. Math. 5, 183-195 (1955).

[5] FrIsch, R. A. K. The Multiplex Method for Linear Programming, Memo. Univ. Inst. of Economics. Oslo, 17. Oktober 1955.

[6] KrelLe, W., und H. P. KÜNZx: Lineare Programmierung. Verlag Industrielle Organisation. Zürich (1958).

[7] KüNZI, H. P.: Die Simplexmethode zur Bestimmung einer Ausgangslösung bei bestimmten linearen Programmen. Unternehmensforsch. 2, 60-69 (1958).

[8] KuHN, H. W., and A. W. Tucker (editors): Linear Inequalities and Related Systems. Annals of Mathematics Studies, No. 38, Princeton Univ. Press, Princeton, N.Y., 1956.

[9] Lemke, C. E.: The Dual Method of Solving the Linear Programming Problem. Nav. Res. Log. Quart. 1, 36- 47 (1954).

[10] МотzкIN, T. S.: Beiträge zur Theorie der Linearen Ungleichungen. Inaugural-Dissertation, Basel 1936.

[11] ZoutendiJk, G.: Methods of Feasible Directions. Elsevier Publishing Company 1960.

Wirtschaftswissenschaftliches Institut der Universität Zürich 